\title{
ARTÍ́CULO
}

\section{La enseñanza de las ciencias sociales} y el tratamiento de la información. Una experiencia con el uso de webquests en la formación del
profesorado de educación primaria

\section{Pedro Miralles Martínez}

pedromir@um.es

Profesor Titular en el Departamento de Didáctica de las Ciencias Matemáticas y Sociales

de la Facultad de Educación de la Universidad de Murcia

\section{Cosme Jesús Gómez Carrasco}

cjgomez@um.es

Profesor Contratado Doctor en el Departamento de Didáctica de las Ciencias Matemáticas y Sociales de la Facultad de Educación de la Universidad de Murcia

\section{Laura Arias Ferrer}

larias@um.es

Profesora Contratada Doctor en el Departamento de Didáctica de las Ciencias Matemáticas y Sociales de la Facultad de Educación de la Universidad de Murcia

Fecha de presentación: mayo de 2012 Fecha de aceptación: abril de 2013 Fecha de publicación: julio de 2013

\section{Cita recomendada}

MIRALLES, Pedro; GÓMEZ, Cosme Jesús; ARIAS, Laura (2013). «La enseñanza de las ciencias sociales y el tratamiento de la información. Una experiencia con el uso de webquests en la formación del profesorado de educación primaria» [artículo en línea]. Revista de Universidad y Sociedad del Conocimiento (RUSC). Vol. 10, n. 2 2. págs. 98-111. UOC. [Fecha de consulta: dd/mm/aa].

$<$ http://rusc.uoc.edu/ojs/index.php/rusc/article/view/v10n2-miralles-gomez-arias/v10n2-mirallesgomez-arias-es $>$

<http://dx.doi.org/10.7238/rusc.v10i2.1536>

ISSN 1698-580X 


\begin{abstract}
Resumen
El objetivo principal de este trabajo es mostrar la utilidad de las tecnologías de la información y de la comunicación en la formación del profesorado de educación primaria. Con el uso de estos recursos, se han elaborado una serie de experiencias, llevadas a cabo con los estudiantes, para facilitar el aprendizaje de los elementos curriculares necesarios en la enseñanza de las ciencias sociales. Esta práctica se ha desarrollado en la asignatura Metodología didáctica para la enseñanza de las ciencias sociales, ofertada en el grado de Educación Primaria. La experiencia muestra cómo los alumnos elaboraron una webquest y el proceso de aprendizaje tanto de los contenidos curriculares de la asignatura como de las competencias desarrolladas.
\end{abstract}

\title{
Palabras clave
}

TIC, didáctica de las ciencias sociales, formación del profesorado, educación primaria

\section{Social sciences teaching and information processing. An experience using WebQuests in primary education teacher training}

\begin{abstract}
The main aim of this study is to show the utility of information and communication technologies (ICTS) in primary education teacher training. A series of experiences using these resources was produced and then undertaken by students to facilitate their learning of the curricular components of social sciences teaching. This practical exercise was implemented on the Didactic Methodology subject for social sciences teaching, offered on the Primary Education bachelor's degree course. The experience shows how the students produced a WebQuest and developed the learning process for both the subject's curricular content and competencies.
\end{abstract}

\section{Keywords}

ICTS, social sciences didactics, teacher training, primary education

\section{Introducción}

La experiencia didáctica que se presenta en este trabajo se llevó a cabo en la asignatura Metodología didáctica para la enseñanza de las ciencias sociales, en el grado de Educación Primaria de la Universidad de Murcia. En ella, los alumnos deben introducirse en la metodología didáctica y en el uso de las herramientas y los recursos necesarios para aplicar, en un aula de primaria, sus conocimientos de ciencias sociales. Es necesario que el alumno sepa manejar la variedad de recursos y herramientas docentes del área, así como las nuevas utilidades que ofrecen las TIC para mejorar la enseñanza.

Teniendo en cuenta las especificaciones concretas de la asignatura, el equipo docente propuso una práctica obligatoria a través de la creación de una webquest para motivar al alumnado en el uso de las nuevas tecnologías, tanto para su propia formación como para su futura práctica docente. Los estudiantes ampliaron aspectos concretos de los contenidos de la asignatura, confeccionando un nuevo material o realizando nuevas aplicaciones de estos contenidos. El alumnado tuvo que ejercitar 
una serie de capacidades: análisis, evaluación, síntesis, argumentación, evaluación de información, etc., Io que le proporcionó estrategias útiles para adquirir habilidades con las TIC y respecto al acceso a la información a través de internet. El elemento clave es el papel activo que los alumnos tuvieron en la creación de recursos (Soep, 2012). No cabe duda de que el aprendizaje a través de las webquests permite trabajar en la adquisición de competencias y habilidades, como el tratamiento de la información y la competencia digital, aprender a aprender, la autonomía e iniciativa personal, entre otras (Barba, 2008). Pero, además, su uso concreto en la enseñanza universitaria se muestra altamente eficaz, tal como demuestran diversos estudios al respecto (Bernabé, 2008; Quintana y Martín, 2011).

Sin embargo, la novedad de nuestra propuesta reside en que el proceso de elaboración corre a cargo de los alumnos, así como en la planificación de la tarea central propuesta para futuros usos educativos. Sin duda, en el ámbito de la enseñanza universitaria, las nuevas tecnologías están infrautilizadas (Selwyn, 2007) y todavía existe un dominio de la clase tradicional, en la que escasea el trabajo colaborativo (Welker y Berardino, 2005-2006). Con esta actividad se buscó superar tales obstáculos y que los estudiantes participaran plenamente en la elaboración de los contenidos de ciencias sociales, además de que fueran conscientes de que un correcto uso y una gestión eficaz de la información son tan importantes como el producto de la propia información.

\section{Las TIC, la formación del profesorado y la enseñanza de las ciencias sociales}

El papel que actualmente ocupan las TIC en la sociedad ha supuesto que el docente haya adquirido un nuevo rol en la gestión y transmisión del conocimiento (González, 2008). Por ello es una cuestión fundamental aprovechar las dos principales funciones de internet (transmitir información y facilitar la comunicación) como soporte didáctico en la enseñanza presencial. A pesar de que la integración de las TIC en la educación suele relacionarse con el uso de nuevos medios y con la innovación educativa, esta realmente debe hacerlo con una mejora significativa del proceso de enseñanza-aprendizaje (De Haro, 2009). Estos cambios pueden representar ajustes más que revoluciones (López Yáñez, 2010), pueden entenderse como un elemento de transformación, renovación y mejora (Rubia y Anguita, Jarrín y Ruiz, 2010), y deben ser cambios planificados e intencionados (Sangrà, 2011). El uso de las nuevas tecnologías no implica automáticamente un aumento de la calidad de la enseñanza. De hecho, la integración de las TIC en modelos formativos no adecuados, en vez de mejorar el aprendizaje de los alumnos, lo empeora, lo que incrementa la carga de docentes y discentes (Rodríguez Izquierdo, 2011). Los futuros docentes, por tanto, deben orientar el uso de las nuevas tecnologías para mejorar el proceso de aprendizaje y fomentar una enseñanza en habilidades, capacidades y competencias.

La introducción de la competencia digital en el ámbito educativo ha provocado que los centros tuvieran que revisar su infraestructura y sus recursos, así como que el profesorado tuviera que ponerse al día en cuanto al uso y la enseñanza de las nuevas tecnologías, para conseguir la alfabetización digital de sus alumnos (Rodríguez Illera, 2004). Por esto es tan importante que los futuros docentes superen algunos de los problemas que surgen para el correcto manejo de las TIC y su aplicación a la 


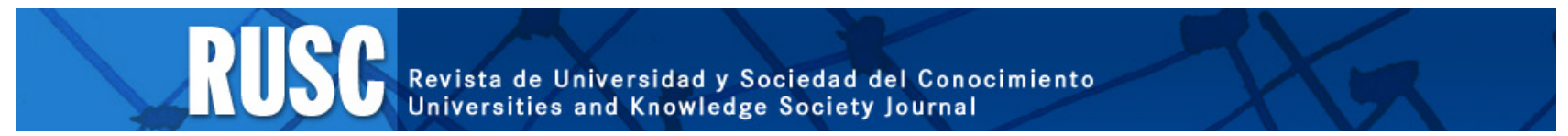

http://rusc.uoc.edu

La enseñanza de las ciencias sociales y el tratamiento de la información...

enseñanza. Tal y como indican Gutiérrez, Yuste, Cubo y Lucero (2011), la falta de habilidades tecnológicas del alumnado y de capacidades en el trabajo colaborativo, o las pobres habilidades lectoras, pueden suponer un inconveniente a la hora de implementar nuevas metodologías de aprendizaje que integren las TIC. A pesar de que los alumnos universitarios actuales conviven con el uso cotidiano de las nuevas tecnologías, son varios los trabajos que alertan de que muchos estudiantes se muestran inseguros respecto a cómo integrar esas nuevas herramientas en su educación (Barnes, Marateo y Ferris, 2007).

Para desarrollar plenamente la competencia en el tratamiento de la información y la competencia digital hay que insistir en que la información en sí no lleva directamente al conocimiento. Transformar la información en conocimiento exige unas destrezas de razonamiento para organizar la información, relacionarla, analizarla, sintetizarla y hacer inferencias y deducciones de distinto nivel de complejidad. Según Contreras y Vásquez (2007), el desarrollo de la indagación implica fortalecer varias habilidades: observación, sistematización de datos, planteamiento de hipótesis, reflexión y acción. Es evidente que entre el profesorado existe una importante convicción de que son necesarios nuevos aprendizajes y nuevas habilidades docentes, ya que la sociedad actual es muy diferente a la de generaciones anteriores (López Facal, 2010). Esto supone que para adquirir esos conocimientos es necesario aprovechar la información que proporcionan las nuevas tecnologías y analizarla de forma crítica mediante el trabajo personal autónomo y el trabajo colaborativo, conociendo y relacionándose con entornos físicos y sociales cada vez más amplios (Coll, Mauri y Onrubia, 2006; Lara y Repáraz, 2007; MacGregor y Lou, 2004).

Todos estos aspectos deben situar al profesorado como protagonista activo en el tratamiento de la competencia digital. Es necesario que el profesor haga que el alumno desarrolle una serie de habilidades que le permita el acceso y la gestión de la información disponible en internet, teniendo en cuenta que la red experimenta una actualización dinámica y permanente (Monereo, 2005). No obstante, para que los profesores puedan transmitir esas habilidades, es imprescindible que las adquiera anteriormente y de forma correcta. Necesitamos un profesorado reflexivo y activo, capaz de generar aprendizajes profundos gracias a la riqueza de los materiales y recursos didácticos que nos proporcionan las TIC (De Pablos, Area, Valverde y Correa, 2010). Por ello en las facultades de Educación debe enfocarse la adquisición de esa competencia más allá de la habilidad como usuario de las nuevas tecnologías, y adentrarse también en la gestión y en el tratamiento de la información. Esto supone que el profesor debe adoptar un nuevo perfil abierto a la innovación, y emplear las nuevas tecnologías y los entornos virtuales para mejorar el proceso de enseñanza-aprendizaje (De Pablos, 2008, Del Moral y Villalustre, 2012).

En la última década, los estudios sobre la enseñanza de las ciencias sociales han insistido en la necesidad de incluir las TIC en el aula. Como opina Acosta (2010), la historia se encuentra en desventaja respecto a las ciencias experimentales, debido a la imposibilidad de reproducir los hechos históricos objeto de estudio. La introducción de las TIC da la oportunidad a los docentes de solventar algunos de estos problemas. Las principales ventajas esgrimidas sobre el uso de las nuevas tecnologías para la enseñanza de las ciencias sociales han girado en torno al aprendizaje autorregulado y significativo, a la colaboración (tanto entre alumnos como entre docentes y discentes) y respecto al hecho de que la enseñanza pueda ser más individualizada. También, como han mostrado Hernàndez (2011) o Rivero 
(2011), la utilización de figuras virtuales y la interactividad que permiten las nuevas tecnologías o el uso de la multimedia expositiva son uno de los principales elementos que potencian la enseñanza de la geografía y la historia.

Sin embargo, también es necesario incidir en la utilidad que poseen las nuevas tecnologías en el ámbito de las ciencias sociales, más allá de ser un recurso de apoyo para la práctica docente, y mostrar la oportunidad que estas nos brindan para potenciar la competencia social y ciudadana. En este sentido, si consideramos, como indica Selwyn (2004), que una persona es competente en valores cívicos cuando es capaz de conocer, hacer y tener una actitud en un contexto determinado de actuación en los planos político, social y económico, el correcto manejo y la gestión de la información que brindan las nuevas tecnologías permitiría a los estudiantes adquirir esas habilidades sociales y ejercer una ciudadanía activa, participativa y crítica. Es necesario que los futuros maestros posean unas competencias docentes que les permitan ejercer su labor formadora en concordancia con los objetivos didácticos planteados desde la nueva normativa (Pavié, 2011). En este sentido, el uso de webquests en la formación del profesorado de educación primaria, desde la didáctica de las ciencias sociales, permite fomentar en los futuros maestros unos conocimientos más profundos de los conceptos clave para la enseñanza de la geografía y la historia. Pero además los ayuda a adquirir, y saber transmitir, habilidades en la gestión y el tratamiento de la información, a lograr una actitud crítica y reflexiva que les permita diferenciar entre hechos y opiniones, así como a afianzar la capacidad de construir conocimiento.

\section{Resultados de la experiencia en el aula}

\subsection{Planteamientos previos}

La actividad descrita la supervisaron los cinco docentes que impartieron dicha asignatura durante el curso 2011-2012, y se realizó en los siete grupos de 3. de grado de Educación Primaria de la Universidad de Murcia, que suman un total de 529 alumnos.

La selección de la webquest respondía a diversos objetivos, que iban más allá del aprendizaje de su creación y uso. Uno era potenciar el aprendizaje autónomo del alumnado mediante el desarrollo de herramientas para un aprendizaje más global y, sobre todo, procedimental y competencial. Esta orientación es la base del carácter innovador del proyecto presentado (González, 2008). Junto a esta, se pretendía que el alumnado adquiriera la capacidad de planificación y programación docente, además de que tomara conciencia de la importancia de la labor de selección y crítica de la información. Esto nos permitía potenciar la reflexión sobre su propio papel como mediador en el proceso de enseñanza-aprendizaje (Marquès, 2011), donde el docente ha de fomentar la construcción del conocimiento, incentivar una actitud crítica y reflexiva, así como promover un aprendizaje que insista en una mayor autonomía del alumno. La webquest resultó una herramienta de gran utilidad para todo ello.

Centrándonos en la aportación de esta propuesta para el trabajo concreto de la planificación docente, destacamos la apreciación que realiza Martín (2004), quien compara el proceso de elaboración de una webquest con el propio de una unidad didáctica convencional. Las partes de las que 
consta son un estímulo para la reflexión sobre la acción docente, lo que contribuye directamente al desarrollo y a la adquisición de la competencia pedagógica y curricular (Correa, 2004). Así se observa al analizar sus apartados:

- Introducción. Se presenta la actividad. Se ofrece un contexto y se diseña un objetivo que ha de ser motivador para el alumnado.

- Tarea. Se especifica la labor que ha de realizar el alumnado. La claridad y la concisión de la propuesta son claves en la redacción del enunciado.

- Proceso. Se describen minuciosamente cada uno de los pasos que ha de seguir el alumnado para poder realizar correctamente la tarea asignada, teniendo en cuenta los problemas que se pueden encontrar durante el proceso.

- Recursos. Para realizar la tarea, el alumno tendrá que valerse de ciertos recursos que podrá encontrar clasificados y comentados en este apartado. Su correcta selección es clave para el éxito de la actividad planteada, ya que han de estar perfectamente adaptados a la cognición de los alumnos a los que se dirige.

- Evaluación. Se señala cómo el docente va a evaluar la actividad, especificando qué se espera obtener de la tarea realizada.

- Conclusiones. Como cierre de la actividad se ha de mostrar una conclusión sobre lo aprendido y/o destacar alguna cualidad de los trabajos.

Además, como complemento a la propuesta anterior, se llevó a cabo, de manera individual y autónoma, una búsqueda de información en la red, lo que llevaba implícito una labor de recopilación, lectura, selección y reflexión. Esto permitió trabajar diversos contenidos propios de las ciencias sociales, recogidos en el currículo de Educación Primaria, y comprobar su nivel de comprensión y capacidad crítica a la hora de adaptar dichos recursos para el ciclo y curso seleccionado. Esta fase se muestra fundamental, ya que la idoneidad del proceso, de la tarea y de los recursos propuestos dependerá del grado de comprensión y dominio del tema seleccionado por parte del creador-organizador de la actividad (Correa, 2004). De esta manera se demandaba del alumnado universitario similares capacidades a las que ellos mismos exigirían a sus futuros alumnos mediante el trabajo de la webquest diseñada: análisis, evaluación, síntesis y argumentación de un grupo de contenidos concretos.

Junto a esto, se planteaba la realización de una guía de la actividad para uso docente, en la que debían indicarse contenidos tales como número de sesiones, objetivos, contenidos y competencias, además del tipo de metodología aplicada. Por último, se concretaba cómo iba a ser evaluada la propuesta y se definían los criterios de evaluación. Con la realización de esta ficha se aseguraba que la planificación de la actividad había sido completamente meditada y se procuraba igualmente el trabajo directo con la legislación educativa vigente.

\subsection{Consideraciones didácticas}

Como se ha señalado, esta actividad perseguía que el alumnado fuera capaz de seleccionar y analizar la información de manera crítica, secuenciar y organizar correctamente objetivos, contenidos, com- 
petencias, actividades, criterios y herramientas de evaluación, así como de evaluar la importancia de la planificación de la acción docente. Además, nos permitía introducir las características propias de la metodología de indagación y facilitaba el aprendizaje y la comprensión del vocabulario específico de la asignatura y de conceptos geográficos e históricos concretos.

De la misma manera, este ejercicio contribuía a la adquisición de gran parte de las competencias genéricas universitarias, especialmente aquellas relacionadas con las habilidades interpersonales, el conocimiento aplicado al campo de estudio, la capacidad para adaptar el conocimiento a la práctica, el trabajo autónomo, las habilidades en la gestión de la información, la capacidad de análisis y síntesis, y las habilidades de investigación (Bernabé, 2008). El propio proceso de creación de la herramienta en sí permite fomentar las habilidades de acceso y gestión de herramientas TIC. La necesidad de incorporar fuentes y recursos fiables y de calidad para completar la tarea de indagación que una webquest conlleva favorece la capacidad de gestión de la información, así como el desarrollo de habilidades de investigación, a la vez que incentiva el pensamiento y análisis crítico. El proceso de planificación de su puesta en práctica es la excusa perfecta para que el estudiante conozca el currículo escolar de ciencias sociales, y la necesidad de evaluar la actividad diseñada permite la reflexión docente sobre la propia actividad.

\subsection{Desarrollo de la actividad}

La elaboración de la webquest se planteó como una actividad grupal (de cuatro a seis miembros). Se desarrolló en tres sesiones prácticas de dos horas cada una, a las que hay que sumar el trabajo autónomo del propio estudiante fuera del aula.

Para su creación se recomendaron dos herramientas, seleccionadas por su facilidad de uso: «El creador de webquest online» (Jorquera, 2011) y el generador «1, 2, 3: tu webquest» (Muñoz y Valero).

Los contenidos en los que trabajó cada grupo de estudiantes se seleccionaban libremente, por lo que las temáticas fueron heterogéneas: ríos de España, conservación del medio ambiente, ciclo del agua, vida de los íberos, descubrimiento de América, entre otros. Esto nos permitió ampliar los contenidos de carácter geográfico e histórico con los que se iba a trabajar en nuestras aulas, a raíz de las exposiciones orales de la actividad. Además, estas iban a ser colgadas en la wiki creada a tal efecto y que facilitaría su consulta. ${ }^{1}$

\subsection{Evaluación de la actividad}

La evaluación de la webquest atendió a unos criterios muy concretos: realismo en su planificación, corrección en la selección de sus contenidos y su originalidad, que eran evaluados mediante un sistema de rúbricas, y que en algunos casos fue acompañado de una actividad de coevaluación, lo que permitió reforzar el proceso de enseñanza-aprendizaje y fomentar el pensamiento crítico (Fernández, 2011).

1. Véase http://didactictac.wikispaces.com/WebQuest. 


\begin{tabular}{|c|}
\hline Criterios de evaluación \\
\hline $\begin{array}{l}\text { Evaluación del proceso de planificación } \\
\square \text { Selecciona, secuencia y organiza correctamente objetivos, contenidos, competencias, metodología y actividades, así como } \\
\text { criterios y herramientas de evaluación. } \\
\square \text { Conoce y utiliza el vocabulario específico referente a la metodología y didáctica de las ciencias sociales. } \\
\square \text { Evalúa la importancia de la planificación en la organización del proceso de enseñanza-aprendizaje. }\end{array}$ \\
\hline $\begin{array}{l}\text { Evaluación de la webquest y sus contenidos } \\
\square \text { Es original y atractiva. } \\
\square \text { Es adecuada para el ciclo/nivel seleccionado. } \\
\square \text { Utiliza un lenguaje y un vocabulario adecuados. } \\
\square \text { Seleccionan información y recursos utilizados de manera crítica. } \\
\square \text { Los recursos seleccionados son adecuados para el ciclo/nivel. } \\
\square \text { Introduce y desarrolla correctamente la tarea y el proceso que ha de realizar el alumno, así como su evaluación y } \\
\text { conclusión. }\end{array}$ \\
\hline
\end{tabular}

El mayor problema al que podíamos enfrentarnos en la puesta en práctica de la propuesta era la inversión de tiempo que podría suponer en el caso de que el desarrollo de la competencia digital del alumnado no fuera el esperado. Por ello, considerábamos necesario conocer la percepción que los alumnos poseían sobre semejante actividad, las dificultades a las que se habían enfrentado y los procedimientos y las actitudes que habían adquirido con su elaboración.

La técnica utilizada para su evaluación fue la de un cuestionario con preguntas cerradas, previamente validado por expertos universitarios del Área de Didáctica de las Ciencias Sociales. Los alumnos lo rellenaron anónimamente; evaluaron un total de doce afirmaciones. La escala de estimación de todas las preguntas fue la siguiente: 1=nada, 2=poco, 3=bastante, 4=mucho. Fue contestado por los 529 alumnos que realizaron la actividad. Los ítems y la media que se obtuvo pueden comprobarse en el cuadro 2.

Al analizar los resultados del cuestionario se comprobó que el alumnado evaluaba positivamente la contribución de esta actividad a la capacidad de programar actividades de manera coherente (3,27 sobre 4), a la comprensión de la importancia de adaptar los recursos de internet a alumnos de diferentes edades y niveles formativos $(3,40)$, a la reflexión sobre el valor de las TIC en el proceso de enseñanza-aprendizaje $(3,24)$ y a valorar la labor del docente que usa las TIC en el aula procurando la innovación educativa $(3,36)$. Igualmente contribuyó a poder comprender la importancia de la planificación en el proceso de enseñanza-aprendizaje $(2,99)$ y a desarrollar un espíritu crítico a la hora de buscar fuentes de información en la red $(2,92)$. En menor medida, la realización de una webquest permitió mejorar las destrezas informáticas de los alumnos (2,50). Pero, en cualquier caso resultó ser una actividad bastante motivadora $(3,06)$ y contribuyó en igual medida a la formación como docentes $(3,04)$ al desarrollar competencias que pueden ser útiles para el futuro laboral $(3,02)$. Finalmente, los estudiantes encuestados se mostraron bastante satisfechos con lo aprendido en la actividad $(3,06)$ y consideraron que la elección de la webquest como recurso fue adecuada $(3,14)$. 
Cuadro 2. Cuestionario de evaluación de la actividad por parte del alumno

\begin{tabular}{|c|c|}
\hline Afirmaciones planteadas & $\begin{array}{c}\text { Valoración } \\
\text { (máx. 4) }\end{array}$ \\
\hline $\begin{array}{l}\text { La realización de la webquest ha contribuido... } \\
\square \text {... a mejorar mis destrezas informáticas. } \\
\square \text {... a comprender la importancia de la planificación en el proceso de e-a. } \\
\square \text {... a programar actividades de manera coherente, teniendo en cuenta objetivos, contenidos, } \\
\text { competencias, etc. } \\
\square \text {.... a desarrollar un mayor espíritu crítico a la hora de buscar fuentes de información en internet. } \\
\square \text {... a comprender la importancia de adaptar los recursos de internet a alumnos de diferentes edades y } \\
\text { niveles formativos. }\end{array}$ & $\begin{array}{l}3,27 \\
2,92\end{array}$ \\
\hline $\begin{array}{l}\text { Además me ha permitido... } \\
\square \text {... reflexionar sobre el valor de las TIC en el proceso de enseñanza-aprendizaje. } \\
\square \text {... evaluar la labor del docente que hace uso de las TIC en el aula, procurando la innovación educativa. }\end{array}$ & $\begin{array}{l}3,24 \\
3,36\end{array}$ \\
\hline $\begin{array}{l}\square \text { Ha resultado una actividad motivadora. } \\
\square \text { Ha contribuido a mi formación como docente. } \\
\square \text { Mi grado de satisfacción con lo aprendido a través de la realización de esta actividad práctica es } \\
\text { satisfactorio. } \\
\square \text { He desarrollado competencias que me pueden ser útiles en mi futuro laboral. } \\
\square \text { La elección de la webquest como recurso sobre el que trabajar es adecuada. }\end{array}$ & $\begin{array}{l}3,06 \\
3,02 \\
3,14\end{array}$ \\
\hline
\end{tabular}

\section{Conclusión}

La elaboración de una webquest y la planificación de su puesta en práctica contribuyó a la formación por competencias. En particular, la importancia adquirida por la competencia relacionada con el tratamiento de la información y la competencia digital exigen del profesorado que tenga habilidades en el correcto uso de la información que proviene de internet, para convertirla en conocimiento, por lo que su formación en este sentido debe ser un elemento principal. Pero esta no puede limitarse al conocimiento del software y su uso en el aula, sino que debe tener siempre como finalidad la mejora del proceso de enseñanza-aprendizaje. Del docente dependerá que se empleen las TIC para una enseñanza tradicional o innovadora.

Los resultados obtenidos de la evaluación que realizaron los 529 alumnos sobre la actividad fueron satisfactorios. El hecho de que el alumnado haya valorado la creación y el trabajo de la webquest en su conjunto, la adquisición de habilidades relacionadas con la programación y la adaptación de actividades y recursos, así como la necesidad de que el docente utilice las TIC para impulsar la innovación educativa, refuerza esta conclusión. La creación de la webquest ha conseguido incentivar la adquisición de nuevos contenidos por parte del alumnado mediante una actividad motivadora, y ha posibilitado a los futuros maestros que se adentren en esas habilidades. El resultado de la experiencia muestra la potencialidad del uso de esta herramienta, tanto en el ámbito didáctico como en el puramente disciplinar, y sus aplicaciones futuras en la práctica docente en el aula. Aun así, somos conscientes de que hay que mejorar algunos aspectos de esta actividad, como, por ejemplo, aquellos relacionados con la adquisición de habilidades que tienen que ver con el desarrollo de un mayor espíritu crítico a la hora de buscar fuentes de información en internet. 
En definitiva, lo importante de la utilización de las nuevas aplicaciones tecnológicas no es usarlas por sí mismas, sino emplearlas para que produzcan innovaciones en la enseñanza. Si no cambia la metodología, las TIC no servirán de mucho. El principal reto estriba en orientarnos hacia un procesamiento social de la información y una construcción colaborativa del conocimiento (en la línea precisamente de las webquests). El uso de estos medios debe fomentar el pensamiento autónomo, creativo y crítico.

\section{Bibliografía}

ACOSTA, Luis M. (2010). «La enseñanza-aprendizaje de la historia en bachillerato y las TIC: la introducción de la estrategia webquest». Clío. N.o 36 [en línea]. [Fecha de consulta: 30 de enero de 2012]. $<$ http://clio.rediris.es>

BARBA, Carme (2008). «La webquest, una metodología de fututo». Quaderns Digitals. N.o 51.

BARNES, Kassandra; MARATEO, Raymond C.; FERRIS [et al.] (2007). «Teaching and learning with the net generation». Innovate: Journal of Online Education. Vol. 3, n. ${ }^{\circ} 4$.

BERNABÉ, lolanda (2008). Las webquests en el Espacio Europeo de Educación Superior (EEES). Desarrollo y evaluación de competencias con tecnologías de la información y la comunicación (TIC) en la universidad [en línea]. [Fecha de consulta: 3 de mayo de 2012]. <http://www.tesisenred.net/bitstream/handle/10803/10367/bernabe.pdf?sequence=1>

CONTRERAS, David; VÁSQUEZ, Nelson (2007). «Competencias ciudadanas para la alfabetización digital». Íber. Didáctica de las Ciencias Sociales, Geografía e Historia. N. 0 53, págs. 63-72.

COLL, César; MAURI, Teresa; ONRUBIA, Javier (2006). «Análisis y resolución de casos-problemas mediante el aprendizaje colaborativo». RUSC. Revista Universidad y Sociedad del Conocimiento. Vol. 3, n. ${ }^{2}$, págs. 29-41.

CORREA, José M. (2004). «El webquest en la enseñanza universitaria: una experiencia en la formación inicial del profesorado». Qurriculum. N.o 17, págs. 171-186.

DE HARO, Juan J. (2009). «Algunas experiencias de innovación educativa». Arbor. Ciencia, pensamiento y cultura. N. ${ }^{\circ}$ CLXXXV, págs. 71-92. <http://dx.doi.org/10.3989/arbor.2009.extran1207>

DE PABLOS, Juan (2008). «Nuevas formas de trabajo en las aulas universitarias con el soporte de las tecnologías de la información y la comunicación». En: M. E. DEL MORAL y R. RODRíGUEZ (coords.). Experiencias docentes y TIC. Barcelona: Editorial Octaedro, págs. 43-58.

DE PABLOS, Juan; AREA, Manuel; VALVERDE, Jesús [et al.] (2010). Políticas educativas y buenas prácticas con TIC. Barcelona: Graó.

DEL MORAL, M. a Esther; VILLALUSTRE, Lourdes (2012). «Didáctica universitaria en la era 2.0: competencias docentes en campus virtuales». RUSC. Revista Universidad y Sociedad del Conocimiento. Vol. 9, n. 1 , págs. $36-50$

FERNÁNDEZ, Antonio (2011). «La evaluación orientada al aprendizaje en un modelo de formación por competencias en la enseñanza universitaria». En: BUJAN, Karmele; REKALDE, Itiziar; ARAMENDI, Pello (coords.). La evaluación de competencias en la educación superior. Sevilla: Eduforma, págs. 37-57. 
GONZÁLEZ, Juan C. (2008). «TIC y la transformación de la práctica educativa en el contexto de las sociedades del conocimiento». Revista de Universidad y Sociedad del Conocimiento. Vol. 5, n. 2, págs. 1-8 [en línea]. [Fecha de consulta: 30 de enero de 2012]. <http://www.uoc.edu/ojs/index.php/rusc/article/view/v5n2-gonzalez>

GUTIÉRREZ, Prudencia; YUSTE, Rocío; CUBO, Sixto [et al.]. (2011). «Buenas prácticas en el desarrollo de trabajo colaborativo en materias TIC aplicadas a la educación». Profesorado. Revista de Curriculum y Formación del Profesorado. Vol. 15, n.`1, págs. 179-194.

HERNÀNDEZ, Francesc X. (2011). «La iconografía en la didáctica de las ciencias sociales». Íber. Didáctica de las Ciencias Sociales, Geografía e Historia. N. 68 , págs. 7-16.

LARA, Sonia; REPÁRAZ, Charo (2007). «Eficacia de las webquest para el aprendizaje cooperativo». Revista Electrónica de Investigación Psicoeducativa. Vol. 5(3), n.` 13, págs. 731-756.

LÓPEZ FACAL, Ramón (2010). «Didáctica para el profesorado en formación: ¿por qué hay que aprender a enseñar ciencias sociales?» Íber. Didáctica de las Ciencias Sociales, Geografía e Historia. N. 05 , págs. 75-82.

LÓPEZ YÁÑEZ, Julián (2010). «La práctica de la innovación educativa y nuestro conocimiento sobre ella». Profesorado. Revista de Currículum y Formación del Profesorado. Vol. 14, n. o 1, págs. 1-5.

MACGREGOR, Kim; LOU, Yiping (2004). «Web-Based Learning: How Task Scaffolding and Website Designed Support Knowledge Acquisition». Journal of Research on Technology in Education. Vol. 37, n. 2 , págs. 161-175

MARQUĖS, Pere (2011). Los docentes hoy: funciones, roles, competencias necesarias en TIC y formación [en línea]. [Fecha de consulta: 30 de marzo de 2012].

$<$ http://peremarques.pangea.org/docentes.htm>

MARTÍN, Ignacio (2004). «La webquest en el área de ciencias sociales: aprendizaje de las TIC en contextos educativos». Íber. Didáctica de las Ciencias Sociales, Geografía e Historia. N. o 41, págs. 77-96.

MONEREO, Carles (2005) (coord.). Internet y las competencias básicas: aprender a cooperar, a comunicarse, a participar, a aprender. Barcelona: Graó.

PAVIÉ, Alex (2011). «Formación docente: hacia una definición del concepto de competencia profesional docente». REIFOP. Vol. 14, n.o 1, págs. 67-80.

QUINTANA, Jordi; MARTíN, M. a Victoria (2011). «Difusión y uso de webquests en el ámbito universitario español». Observatorio de la Educación Digital-Universidad de Barcelona. [en línea]. [Fecha de consulta: 20 de enero de 2012]

<http://oed.ub.edu/PDF/Informe_WebQuest_castellano.pdf>

RIVERO, Pilar (2011). «Un estudio sobre la efectividad de la multimedia expositiva para el aprendizaje de la historia». Enseñanza de las Ciencias Sociales. Revista de investigación. N. o 10, págs. 45-50.

RODRÍGUEZ ILLERA, José Luis (2004). «Las alfabetizaciones digitales». Bordón. Vol. 56, n.`3-4, págs. $431-441$.

RODRÍGUEZ IZQUIERDO, Rosa María (2011). «Repensar la relación entre las TIC y la enseñanza universitaria: problemas y soluciones». Profesorado. Revista de Currículum y Formación del Profesorado. Vol. 15, n. 1 , págs. 9-22.

RUBIA, Bartolomé; ANGUITA, Rocío; JARRÍN, Iván [et al.] (2010). «Los procesos de innovación educativa en la formación universitaria, nuevos generadores de buenas prácticas en tecnología educativa». 
Revista Electrónica Teoría de la Educación: Educación y Cultura en la Sociedad de la Información. Vol. 11, n. ${ }^{1}$, págs. 96-120.

SANGRÀ, Albert (2011). «Estratègies, accions i fases dels processos d'integració de les TIC en la innovació docent universitària». Aloma. Revista de Psicologia, Ciències de l'Educació i de l'Esport. N.o 29, págs. 291-306.

SELWYN, Neil (2004). Literature Review in Citizenship, Technology and Learning. Bristol: Futurelab.

SELWYN, Neil (2007). «The use of computer technology in university teaching and learning: a critical perspective». Journal of Computer Assisted Learning. N. 23, págs. 83-94. <http://dx.doi.org/10.1111/j.1365-2729.2006.00204.x>

SOEP, Elisabeth (2012). «Generación y recreación de contenidos digitales por los jóvenes: implicaciones para la alfabetización mediática». Comunicar. Revista Científica de Comunicación y Educación. N. 38, págs. 93-100.

WELKER, J.; BERARDINO, L. (2005-2006). «Blended learning: Understanding the middle ground between traditional classroom and fully online instruction». Journal of Educational Technology Systems. Vol. 34, n.o 1, págs. 33-55.

$<$ http://dx.doi.org/10.2190/67FX-B7P8-PYUX-TDUP>

\section{Recursos web}

JORQUERA, Miguel Ángel (2011). El creador de webquest online. Consejería de Educación de la Región de Murcia.

$<$ www.cepdeorcera.org/majwq>

MUÑOZ, Francisco; VALERO, Alejandro. 1, 2, 3: tu webquest.

$<$ www.aula21.net/Wqfacil/webquest.htm> 


\section{Sobre los autores}

Pedro Miralles Martínez

pedromir@um.es

Profesor Titular en el Departamento de Didáctica de las Ciencias Matemáticas y Sociales de la Facultad de Educación de la Universidad de Murcia

Ha trabajado en todos los niveles educativos, como maestro, profesor y catedrático de instituto y asesor permanente de formación del profesorado. Actualmente es profesor titular de Universidad de Didáctica de las Ciencias Sociales en la Universidad de Murcia, donde coordina un máster y un doctorado sobre investigación e innovación educativa en educación infantil y primaria. Ha dirigido varios proyectos de innovación e investigación educativa sobre didáctica de la historia en educación infantil, primaria y secundaria. Ha participado en diversos proyectos de innovación en la enseñanza universitaria. Es autor de libros y artículos sobre los conceptos de historiografía, patrimonio, familia, ciudadanía, uso de las TIC y evaluación en la enseñanza de la historia, así como sobre el uso de los libros de texto en las aulas.

Cosme Jesús Gómez Carrasco

cjgomez@um.es

Profesor Contratado Doctor en el Departamento de Didáctica de las Ciencias Matemáticas y Sociales de la Facultad de Educación de la Universidad de Murcia

Es Profesor Contratado Doctor en el Departamento de Didáctica de las Ciencias Matemáticas y Sociales de la Facultad de Educación de la Universidad de Murcia. Tras realizar la tesis doctoral en la Universidad de Castilla-La Mancha y ser investigador posdoctoral en la École des Hautes Etudes en Sciences Sociales de París y en el Instituto de Historiografía de la Universidad Carlos III de Madrid, actualmente centra sus líneas de investigación en la evaluación, en el desarrollo de las competencias básicas desde el área de ciencias sociales y en la formación del profesorado de educación primaria. 


\section{DISE \\ Universities and Knowledge Society Journal}

http://rusc.uoc.edu

La enseñanza de las ciencias sociales y el tratamiento de la información...

\section{Laura Arias Ferrer}

larias@um.es

Profesora Contratada Doctor en el Departamento de Didáctica de las Ciencias Matemáticas y Sociales de la Facultad de Educación de la Universidad de Murcia

Licenciada en Historia por la Universidad de Murcia, y doctora por la Universidad de Alicante, actualmente es Profesora Contratada Doctor en el Departamento de Didáctica de las Ciencias Matemáticas y Sociales de la Facultad de Educación de la Universidad de Murcia. Previamente pudo desarrollar su labor como docente e investigadora en la Universidad de Murcia, merced a una beca de investigación predoctoral y a un posterior contrato posdoctoral. Su vinculación con el campo de la didáctica se vio reforzado con su estrecha colaboración con los departamentos de didáctica del Museo de Arte Ibérico del Cigarralejo (Mula, Murcia) y del Museo Arqueológico de Murcia, resultado de la cual fue la elaboración de diversos materiales didácticos. Actualmente centra su trabajo en innovación en la práctica docente y en la formación del profesorado de educación primaria.

Facultad de Educación

Universidad de Murcia

Campus Universitario de Espinardo

30100 Murcia

España

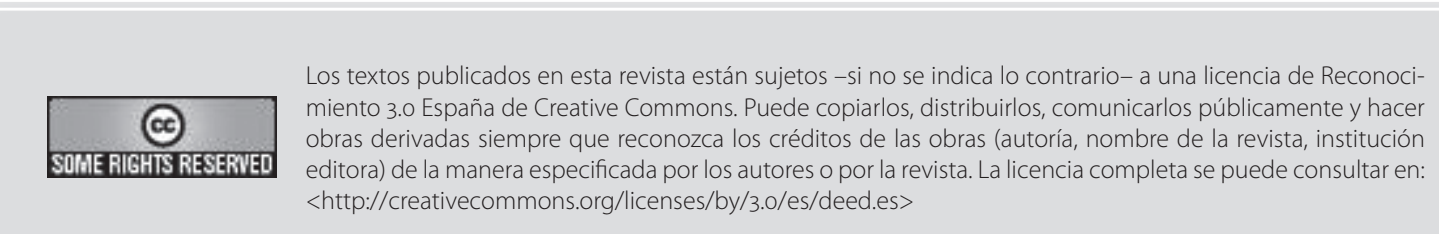

\section{$\bullet$ UOC}

Universitat Oberta

de Catalunya 